\title{
A Novel Transgenic Rat Model of Robust Cerebral Microvascular Amyloid with Prominent Vasculopathy
}

Judianne Davis, ${ }^{*}$ Feng $\mathrm{Xu},{ }^{*}$ Joshua Hatfield, ${ }^{*}$ Hedok Lee, ${ }^{\dagger}$ Michael D. Hoos, ${ }^{\ddagger}$ Dominique Popescu, ${ }^{\S}$ Elliot Crooks,,${ }^{\ddagger}$ Regina Kim, ${ }^{*}$ Steven 0. Smith, ${ }^{\ddagger}$ John K. Robinson, ${ }^{*}{ }^{\mp}$ Helene Benveniste, ${ }^{\dagger}$ and William E. Van Nostrand ${ }^{* \|}$

From the George and Anne Ryan Institute for Neuroscience* and the Departments of Psychology "and Biomedical and Pharmaceutical Sciences," University of Rhode Island, Kingston, Rhode Island; the Department of Anesthesiology, ${ }^{\dagger}$ Yale University, New Haven, Connecticut; and the Departments of Biochemistry and Cell Biology ${ }^{\ddagger}$ and Psychology, ${ }^{\S}$ Stony Brook University, Stony Brook, New York

\author{
Accepted for publication \\ July $31,2018$. \\ Address correspondence to \\ William E. Van Nostrand, \\ Ph.D., George and Anne Ryan \\ Institute for Neuroscience, \\ University of Rhode Island, 130 \\ Flagg Rd., Kingston, RI \\ 02881. E-mail: \\ wvannostrand@uri.edu.
}

\begin{abstract}
Accumulation of fibrillar amyloid $\beta$ protein in blood vessels of the brain, a condition known as cerebral amyloid angiopathy (CAA), is a common pathology of elderly individuals, a prominent comorbidity of Alzheimer disease, and a driver of vascular cognitive impairment and dementia. Although several transgenic mouse strains have been generated that develop varying levels of CAA, consistent models of associated cerebral microhemorrhage and vasculopathy observed clinically have been lacking. Reliable preclinical animal models of CAA and microhemorrhage are needed to investigate the molecular pathogenesis of this condition. Herein, we describe the generation and characterization of a novel transgenic rat (rTg-DI) that produces low levels of human familial CAA Dutch/Iowa E220/D23N mutant amyloid $\beta$ protein in brain and faithfully recapitulates many of the pathologic aspects of human smallvessel CAA. rTg-DI rats exhibit early-onset and progressive accumulation of cerebral microvascular fibrillar amyloid accompanied by early-onset and sustained behavioral deficits. Comparable to CAA in humans, the cerebral microvascular amyloid in rTg-DI rats causes capillary structural alterations, promotes prominent perivascular neuroinflammation, and produces consistent, robust microhemorrhages and small-vessel occlusions that are readily detected by magnetic resonance imaging. The rTg-DI rats provide a new model to investigate the pathogenesis of small-vessel CAA and microhemorrhages, to develop effective biomarkers for this condition and to test therapeutic interventions. (Am J Pathol 2018, 188: 2877-2889; https://doi.org/10.1016/j.ajpath.2018.07.030)
\end{abstract}

Cerebral amyloid angiopathy (CAA) is a common cerebral small-vessel disease that involves the accumulation of amyloid $\beta$ protein $(A \beta)$ primarily in small- and medium-sized arteries and arterioles of the meninges and cerebral cortex as well as along the capillaries of the cerebral microvasculature. ${ }^{1-6} \mathrm{CAA}$ has been shown to be present, in varying degrees, in nearly $80 \%$ of elderly individuals. ${ }^{7,8}$ With the involvement of $\mathrm{A} \beta$, it is not surprising that CAA is a common vascular comorbidity in patients with Alzheimer disease (AD). ${ }^{3,7-9}$ Even when controlling for confounders, including $\mathrm{AD}, \mathrm{CAA}$ is a significant contributor to vascular-mediated cognitive impairment and dementia (VCID) and is independently associated with worse cognition and dementia. ${ }^{7,8,10-13}$ In addition to the sporadic cases of CAA and the prominent
CAA that is observed in $\mathrm{AD}$, several monogenic, familial forms of CAA exist that result from mutations that reside within the $\mathrm{A} \beta$ peptide sequence of the $A P P$ gene, including the Dutch E22Q and Iowa D23N variants. ${ }^{12-14}$

CAA can present as two prominent forms, known as CAA type 1 and CAA type $2 .{ }^{6}$ In CAA type 2 , the amyloid deposition is largely restricted within the vessel wall of the

Supported by NIH grants NS091969 (W.E.V.N., J.K.R., and H.B.) AG053991 (H.B. and W.E.V.N.), NS092696 (W.E.V.N. and S.O.S.), and AG027317 (W.E.V.N. and S.O.S.).

Disclosures: None declared.

A guest editor acted as the Editor-in-Chief for this article. No person at Stony Brook University was involved in the peer review or final disposition of this article. 
meningeal and cortical arterioles and generally does not promote perivascular neuroinflammation. ${ }^{5,6,15}$ In contrast, CAA type 1 involves amyloid deposition along transitional cerebral microvessels and capillaries and is present in nearly half of AD cases. ${ }^{5,6}$ In contrast to larger-vessel CAA type 2, in which the amyloid is largely confined within the vessel wall, CAA type 1 results in penetrance of the fibrillar amyloid deposits into the surrounding brain parenchyma-so-called dyshorric amyloid - a feature that promotes a strong perivascular neuroinflammatory response. ${ }^{5,6,16,17}$ In particular, cerebral microvascular amyloid deposition is often correlated with worse cognition and dementia in individuals afflicted with AD and sporadic/familial CAA disorders. ${ }^{18-22}$

Transgenic mice, which robustly overexpress human $A \beta$ protein precursor $(\mathrm{A} \beta \mathrm{PP})$ in brain and develop cerebral $\mathrm{A} \beta$ deposits, have been a significant advancement for the study of the pathogenic effects of brain $A \beta$ accumulation in vivo. Several well-established transgenic mouse lines have been developed that overproduce human $A \beta$ peptides in brain that develop age-dependent accumulation of parenchymal $A \beta$ deposits with varying degrees of CAA and associated vasculopathies. ${ }^{23-26}$ Specifically, transgenic mouse strains were developed that express human $\mathrm{A} \beta \mathrm{PP}$ in brain, harboring one or more of the familial CAA mutations. ${ }^{27,28}$ More important, these familial CAA transgenic mouse lines revealed the specific role of CAA in neuroinflammation and VCID. ${ }^{29-32}$ However, as with all mouse models, CAA transgenic mice possess some significant limitations to more fully recapitulate and study the equivalent human condition. In particular, consistent and robust microbleeds have been elusive in most CAA mouse models. Therefore, more appropriate animal models of small-vessel CAA are necessary to progress our understanding of the pathogenesis and its contribution to structural changes in the brain and VCID.

Herein, we describe generation and characterization of a new transgenic rat model (rTg-DI) for small-vessel CAA. rTg-DI rats exhibit early-onset and progressive accumulation of cerebral microvascular fibrillar amyloid accompanied by behavioral deficits. Fibrils seeded from the vascular amyloid isolated from rTg-DI rats exhibit intense bands in Fourier transform infrared (FTIR) spectra characteristic of antiparallel $\beta$-sheet, similar to that previously observed for human cerebral vascular amyloid. ${ }^{33}$ Moreover, in rTg-DI rats, the cerebral microvascular $A \beta$ deposits cause capillary structural changes, promote prominent perivascular glial activation, and produce consistent, robust microhemorrhages with small-vessel occlusions that are readily detected by magnetic resonance imaging (MRI).

\section{Materials and Methods}

\section{Generation of Transgenic Rats}

All work with animals followed NIH guidelines and was approved by the University of Rhode Island (Kingston, RI), Yale University (New Haven, CT), and Stony Brook
University (Stony Brook, NY) Institutional Animal Care and Use Committees. A pcDNA3 vector containing $2.1 \mathrm{~kb}$ of human APP (isoform 770) cDNA was used to introduce the mutations Swedish K670N/M671L, Dutch E693Q, and Iowa D694N using the QuikChange kit (Stratagene, La Jolla, CA). The APP770-SwDI cDNA was amplified by PCR using primers containing the NheI five linker and SacII three linker. The PCR product was digested and subcloned between exons II and IV of a Thy1.2 expression cassette using NheI and SacII restriction sites. The completed construct was entirely sequenced to confirm its integrity. The 9-kb transgene was liberated by NotI/PvuI digestion, purified, and microinjected into pronuclei of SpragueDawley single-cell embryos. Founder rats were identified by Southern blot analysis of tail DNA. Transgenic offspring were determined by PCR analysis of tail DNA using the following primers specific for human A $\beta P P$ : $5^{\prime}$-AGGACTGACCACTCGACCAG-3' (forward) and 5'-CGGGGGTCTAGTTCTGCAT- $3^{\prime}$ (reverse), generating a 370 bp product. All subsequent analyses were performed with heterozygous transgenic rats.

\section{Immunoblot Quantitation of $\mathrm{A} \beta \mathrm{PP}$}

Rat forebrain tissues were homogenized in 10 volumes of 50 $\mathrm{mmol} / \mathrm{L}$ tris- $\mathrm{HCl}(\mathrm{pH} 7.5$ ) containing $150 \mathrm{mmol} / \mathrm{L} \mathrm{NaCl}, 1 \%$ SDS, $0.5 \%$ Nonidet P-40, $5 \mathrm{mmol} / \mathrm{L}$ EDTA, and proteinase inhibitor mixture (Roche Applied Science, Indianapolis, IN). The tissue homogenates were clarified by centrifugation at $14,000 \times g$ for 10 minutes. Protein concentrations of the resulting supernatants were determined using the bicinchoninic acid protein assay kit (Fisher Scientific, Houston, TX). The levels of $\mathrm{A} \beta \mathrm{PP}$ in the forebrain tissue homogenates were determined by performing quantitative immunoblotting, as previously described. ${ }^{27}$ Samples were probed with either monoclonal antibody $\mathrm{P} 2-1$, which is specific for human $\mathrm{A} \beta \mathrm{PP},{ }^{34}$ or monoclonal antibody $22 \mathrm{C} 11$ (Chemicon International, Inc., Temecula, $\mathrm{CA}$ ), which detects both rat and human $\mathrm{A} \beta \mathrm{PP}$. Bands corresponding to A $\beta \mathrm{PP}$ were measured using a LI-COR imaging system (LI-COR Biosciences, Lincoln, NE).

\section{Quantitation of $A \beta$ Peptides}

Soluble pools of $A \beta 40$ and $A \beta 42$ were determined by performing specific enzyme-linked immunosorbent assays (ELISAs) on carbonate-extracted rat forebrain tissue, and subsequently the insoluble $A \beta 40$ and $A \beta 42$ levels were determined by ELISA of guanidine lysates of the insoluble pellets, resulting from the carbonate-extracted rat brain tissue, as previously described ${ }^{35,36}$ In the sandwich ELISAs, $A \beta 40$ and $A \beta 42$ were captured using their respective carboxyl-terminal specific antibodies, monoclonal antibody (mAb) $2 \mathrm{G} 3$ and $\mathrm{mAb} 21 \mathrm{~F} 12$; biotinylated m3D6, specific for human $\mathrm{A} \beta$, was used for detection (kindly provided by Lilly Research Laboratories, Indianapolis, IA). ${ }^{35}$ Cerebral microvessels were isolated from rat forebrains, as previously described. ${ }^{27,37}$ The levels of 
cerebral microvascular $\mathrm{A} \beta 40$ and $\mathrm{A} \beta 42$ were measured in guanidine lysates of the brain microvessels isolated from rTg-DI rats. Each rat brain or microvessel lysate was measured in triplicate and compared with linear standard curves generated with known concentrations of human $A \beta$ using a Spectramax M2 plate reader (Molecular Devices, Sunnyvale, CA).

\section{Isolation of Cerebral Capillary Amyloid Seeds}

Brain capillaries were isolated from the cortices of 12-month-old rTg-DI rats, as previously described. ${ }^{27,37}$ The isolated microvessels were analyzed microscopically by staining with thioflavin $\mathrm{S}$ and immunolabeling with an antibody to collagen IV to confirm the isolation of amyloidcontaining microvessels. The remaining microvessels were treated with $3 \mathrm{mg} / \mathrm{mL}$ collagenase at $37^{\circ} \mathrm{C}$ overnight. The collagenase-treated samples were centrifuged at 16,000 $\times g$ for 5 minutes, and the resulting pellet was washed twice with tris-buffered saline. The amyloid pellet was resuspended in tris-buffered saline and again analyzed microscopically by staining with thioflavin $\mathrm{S}$ and immunolabeling with an antibody to collagen IV to confirm the isolation of vascular amyloid deposits and the absence of microvessels.

\section{Seeding of Fibrils from Vascular Amyloid}

For the preparation of fibrils from isolated vascular amyloid, isolated vascular amyloid was sonicated for using a Fisher Scientific tip sonicator at $30 \%$ power in four 10 -second bursts. Monomeric (synthetic) A $\beta 40$-wild-type peptide was added to the seeds in three steps at 1-hour intervals. The A $\beta 40$-wildtype peptide was synthesized using N-t-Boc chemistry on an ABI 430A solid-phase peptide synthesizer (ERI Amyloid Laboratory, Oxford, CT) and purified by high-performance liquid chromatography. On the basis of analytical reversephase high-performance liquid chromatography, the peptide purity was $>98 \%$. The mass of the purified peptide was measured using matrix-assisted laser desorption or electrospray ionization mass spectrometry and was consistent with the calculated mass for the peptide. The peptides were isotopically labeled with $1-{ }^{13} \mathrm{C}$ at Leu17, Ala21, Gly33, and Gly37 for FTIR measurements. Purified, lyophilized A $\beta$ peptides were aliquoted in hexafluoroisopropanol and freeze dried for at least 4 days with a vacuum of 25 mTorr. For preparing monomeric $\mathrm{A} \beta$, the peptide was dissolved in $50 \mathrm{mmol} / \mathrm{L}$ $\mathrm{NaOH}$ at a concentration of $2.2 \mathrm{mmol} / \mathrm{L}$, then diluted in 10 $\mathrm{mmol} / \mathrm{L}$ phosphate buffer $(\mathrm{pH} 7.4)$ at low temperature $\left(4^{\circ} \mathrm{C}\right)$. The $A \beta$ solutions were then filtered with a $0.2-\mu \mathrm{m}$ cellulose acetate filter to remove insoluble aggregates. The $A \beta$ concentration was determined by the absorption at $270 \mathrm{~nm}$ using a molar extinction coefficient of $\varepsilon=1405 / \mathrm{cm} \cdot \mathrm{mol} / \mathrm{L}$.

\section{Fluorescence Spectroscopy}

Thioflavin $\mathrm{T}$ fluorescence measurements were performed on a SpectraMax M2 spectrofluorimeter plate reader. The $A \beta$ peptide concentration was $100 \mu \mathrm{mol} / \mathrm{L}$, and the thioflavin T concentration was $50 \mu \mathrm{mol} / \mathrm{L}$. Thioflavin $\mathrm{T}$ fluorescence was monitored every 15 seconds with excitation, emission, and automatic cutoff wavelengths of 446,490 , and $475 \mathrm{~nm}$, respectively.

\section{FTIR Spectroscopy}

FTIR spectra were obtained on a Bruker Vertex 70 spectrometer using a germanium attenuated total reflection plate with a spectral resolution of $4 / \mathrm{cm}$. Samples were prepared by drying 50 to $100 \mu \mathrm{L}$ of peptide solution on the plate surface.

\section{Tissue Preparation}

Rats were euthanized at designated time points and perfused with cold phosphate-buffered saline (PBS); forebrains were removed and dissected through the midsagittal plane. One hemisphere was immersion fixed with $70 \%$ ethanol overnight and subjected to increasing sequential dehydration in ethanol, followed by xylene treatment and embedding in paraffin. Alternatively, brains were fixed with $4 \%$ paraformaldehyde overnight at $4{ }^{\circ} \mathrm{C}$ and subjected to increasing concentrations $(10 \%, 20 \%$, and $30 \%)$ of sucrose in PBS, then embedded in OCT compound (Sakura Finetek Inc., Torrance, CA) and snap frozen in dry ice. Sagittal sections were cut $(10 \mu \mathrm{m}$ thick) using a Leica RM2135 microtome (Leica Microsystems Inc., Bannockburn, IL), placed in a flotation water bath at $40^{\circ} \mathrm{C}$, and then mounted on Colorfrost/Plus slides (Fisher Scientific, Houston, TX). In some cases, coronal sections were cut ( $20 \mu \mathrm{m}$ thick) from frozen brains using a Leica CM1900 cryostat (Leica Microsystems Inc.) stored in PBS with $0.02 \%$ sodium azide at $4{ }^{\circ} \mathrm{C}$.

\section{Immunohistochemical Analysis}

Antigen retrieval was performed by treating the tissue sections with $0.2 \mathrm{mg} / \mathrm{mL}$ proteinase $\mathrm{K}$ for 10 minutes at $22^{\circ} \mathrm{C}$. Primary antibodies were detected with Alexa Fluor 594-conjugated donkey anti-rabbit or Alexa Fluor 488-conjugated goat anti-mouse secondary antibodies (1:1000). Staining for fibrillar amyloid was performed using either Amylo-Glo, as described by the manufacturer (Biosensis Inc., Thebarton, SA, Australia), or thioflavin S. The following antibodies were used for immunohistochemical analysis: $m A b$ 66.1 (1:250), which recognizes residues 1 to 5 of human $A \beta^{38} ;$ mAb $2 B 4$ to detect $A \beta 42(1: 1000)$ and rabbit polyclonal antibody to detect $\mathrm{A} \beta 40$ (1:200; Biosource, Camarillo, CA); rabbit polyclonal antibody to collagen type IV to visualize cerebral microvessels (1:100; ThermoFisher, Rockford, IL); and rabbit polyclonal antibodies to glial fibrillary acidic protein (GFAP; 1:200; Dako, Santa Clara, CA) and ionized calcium-binding adapter molecule 1 (Iba-1; 1:200; Fujifilm Wako Pure Chemical, Osaka, Japan) for detection of astrocytes and microglia, 
respectively. Prussian blue iron staining was performed to detect hemosiderin deposits reflecting signs of previous microhemorrhage. ${ }^{39,40}$ Von Kossa calcium staining was used to detect small-vessel occlusion/calcifications in the brain. ${ }^{41}$ In this case, tissue sections were counterstained with pararosaniline.

\section{Quantitative Analysis of Vascular Pathologies}

The percentage area amyloid coverage of cerebral microvessels, the percentage area iron staining, and the numbers of occluded/calcified vessels in the frontotemporal cortex, thalamic, and hippocampal regions were determined in rats at each of the specified ages using stereological principles, as previously described. ${ }^{42}$ Cerebral microvessel tortuosity in different brain regions was determined by immunolabeling the vessels in brain tissue sections, measuring both the traced actual vessel length and the direct straight line, endto-end vessel length, and dividing the actual length/direct length.

\section{Novel Exploration Behavioral Task}

A novel exploration paradigm was included to measure perceptual slowing, as represented in a slowed rate of stimulus encounters. This was a common deficit observed in the similar Tg-SwDI mouse model. ${ }^{31}$ Typically, animals were placed in the open field apparatus to explore four novel and distinct objects for a 5-minute trial period. Interaction with an object was scored by the AnyMaze software version 4.99 (Stoelting, Wood Dale, IL) as entering a predetermined zone surrounding the object.

\section{Magnetic Resonance Imaging Analysis}

All rats were deeply anesthetized with ketamine/xylazine $(75 / 10 \mathrm{mg} / \mathrm{kg}$ ) intraperitoneally, followed by transcardial perfusion fixation. First, vascular perfusion was conducted with $0.1 \%$ heparin/PBS $(20 \mathrm{~mL} /$ minute for 5 minutes $)$, followed by $5 \%$ Magnevist in $10 \%$ buffered formalin solution $(120 \mathrm{~mL} / \mathrm{rat}$ at $20 \mathrm{~mL} /$ minute $)$ using a Pharmacia LKB P-1 peristaltic pump (Pharmacia LKB Biotechnology, Piscataway, NJ). After perfusion fixation of the 12-month-old male rTg-DI and wild-type rats, the brains were removed from the skull with great care so as to not damage the brain parenchyma. The brain specimens were immersed in PBS for $\geq 4$ days. All MRI acquisitions were performed on a Bruker 9.4T/30 magnet (Bruker BioSpin, Billerica, MA) with BGA-12SHP imaging gradient system interfaced to an Avance III console controlled by Paravision 5.1 software (Bruker BioSpin). For the post-mortem highresolution scanning, a cryogenically cooled radiofrequency coil was used as a transmitter and receiver. The brain specimens were imbedded in fomblin (HT230-7K, Fluids and Greases; Kurt J. Lesker, Jefferson Hills, PA) and underwent MRI using a three-dimensional gradient echo sequence with the following imaging parameters: repetition time/echo time/flip angle $=100$ milliseconds $/ 7-30$ milliseconds/25 degrees.

The number of excitations $=1$ resolution $=$ $0.08 \times 0.08 \times 0.08 \mathrm{~mm}$. The brain specimens were subsequently processed and stained for hemosiderin deposits, as described above. The stained whole-brain slices were digitized using an optical microscope (All-in-One Fluorescence Microscope BZ-X700; Keyence Corporation of America, Elmwood Park, NJ), allowing the entire brain slice to be used for matching with the corresponding high-resolution post-mortem MRIs. The optical resolution achieved on the digitized brain slices was $0.377 \mu \mathrm{m} /$ pixel.

The MRIs were displayed as isotropic three-dimensional volumes using Amira software version 6.2 (Thermo Fisher Scientific, Houston, TX). Three-dimensional MRIs, acquired with echo times of 7 and 18 milliseconds, were used for the matching process. The process to match the MRIs to the corresponding histologic brain slice involved several computational steps; and in all processing, the histologic brain slice served as the master template or reference, and the three-dimensional MRI was manipulated to match it. First, three to five histologic slices of interest were chosen, which included thalamic iron staining-positive areas of microhemorrhages. Using the slice feature in Amira, the MRI slice plane that best matched the histologic slice (on the basis of anatomical landmarks, such as the shape of the hippocampus, the corpus callosum, the fimbria, and the shape of the ventral surface) was defined. Using rigid matching (scaling in $x$ - and $y$-axis directions), the MRI was matched to the histologic slice. Finally, the histologic slice was overlaid onto the matched two-dimensional MRI to determine the MRI signatures associated with the iron stain-positive areas.

\section{Statistical Analysis}

Histologic and biochemical data were analyzed by $t$-test at the 0.05 significance level. Behavioral testing data were analyzed by $t$-tests, and the performance of each subject for each testing day was the average latency of the two trials.

\section{Results}

Low-Level Expression of Human CAA Mutant A $\beta P P$ in Brain Results in Progressive and Extensive Microvascular CAA

To target production of human chimeric Dutch/Iowa CAA mutant in neurons of rat brain, an approach used in the previous generation of Tg-SwDI mouse strain was adopted. ${ }^{27}$ The neuronal-specific Thy 1.2 expression cassette was used to drive expression of human $A P P$ harboring the familial AD Swedish K670N/M671L mutations and the familial CAA Dutch $E 693 Q$ and Iowa D694N mutations in neurons in the rat brain. The Swedish A $\beta \mathrm{PP}$ mutations were 
included solely to enhance $\beta$-secretase processing and production of $\mathrm{A} \beta$ peptides. ${ }^{43}$ The familial CAA Dutch and Iowa mutations were included in the transgene to yield chimeric Dutch/Iowa CAA mutant $\mathrm{A} \beta$ peptides in brain, which exhibit markedly enhanced fibrillogenic and vascular pathogenic properties. ${ }^{44,45}$ The transgenic rats (rTg-DI) were generated by microinjection of the human APP770SwDI construct into oocytes in a pure Sprague-Dawley background. The presence of the human A $\beta \mathrm{PP}$ transgene was confirmed in founder and subsequent offspring rats by PCR analysis. All rTg-DI rats used in the subsequent characterization experiments were heterozygous for the human $\mathrm{A} \beta \mathrm{PP}$ transgene.

Two-month-old rTg-DI rats were evaluated for expression of the human A $\beta P P$ transgene using the human A $\beta$ PPspecific monoclonal antibody P2-1. ${ }^{34}$ Immunohistochemical analysis presented in Figure 1, A-F, shows that human A $\beta \mathrm{PP}$ expression was evident throughout the cortex, hippocampus, and thalamus and was restricted to neurons. Immunoblot analysis using mAb P2-1 confirmed that human $\mathrm{A} \beta \mathrm{PP}$ transgene expression was only observed in rTg-DI rats (Figure 1G). Subsequent quantitative immunoblot analysis using the total (rat + human) A $\beta P P$-specific mAb $22 \mathrm{C} 11$ showed that human transgene A $\beta \mathrm{PP}$ expression was modest and only approximately 50\% higher than the levels of endogenous rat $\mathrm{A} \beta \mathrm{PP}$ seen in wild-type rats (Figure $1 \mathrm{H}$ ).

The accumulation of human $A \beta$ peptides was measured in rTg-DI brains as they aged over 12 months. A sandwich ELISA format was used that detected only human $A \beta$ peptides. ${ }^{35,36}$ As the rTg-DI rats aged from 3 to 12 months, there was a progressive increase in the amounts of soluble $\mathrm{A} \beta 40$ in brain, whereas the amounts of soluble $\mathrm{A} \beta 42$ remained fairly constant over this time period (Figure 1I). Most soluble A $\beta$ peptides ( $>90 \%)$ at each age were the shorter $A \beta 40$ isoform. On the other hand, there was a progressive accumulation of both insoluble $A \beta 40$ and $A \beta 42$ as the rats aged (Figure $1 \mathrm{~J}$ ). Again, the most accumulated insoluble $A \beta$ peptide was the shorter $A \beta 40$ isoform. These findings indicate that despite the low level of transgene human $\mathrm{A} \beta \mathrm{PP}$ expression, there is an early-onset and progressive accumulation of Dutch/Iowa CAA mutant $A \beta$ peptides in the brains of rTg-DI rats.

\section{Early-Onset and Extensive Accumulation of Cerebral Microvascular Amyloid Is Associated with Behavioral Impairments and Exhibits a Characteristic Antiparallel Alignment}

Because insoluble levels of cerebral $A \beta$ markedly increase with age in rTg-DI rats, the compartmental distribution of fibrillar amyloid was examined over time. As early as 3 months of age, there was notable accumulation of cerebral microvascular amyloid in the cortical, hippocampal, and thalamic regions of the brain (Figure 2, A-I). Furthermore, as the rTg-DI rats aged to 12 months, there was a dramatic
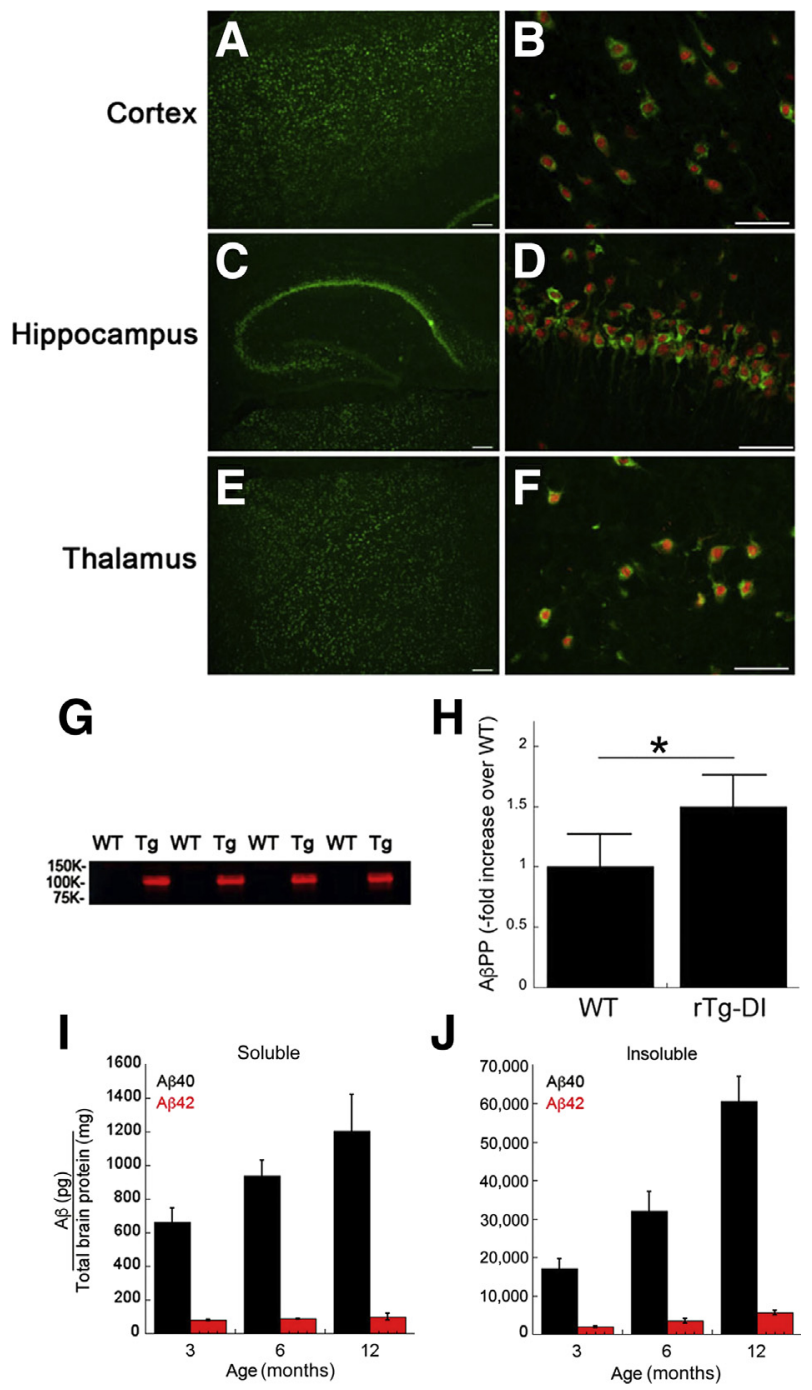

Figure 1 Analysis of transgenic human ABPP expression and progressive $A \beta$ accumulation in $\mathrm{rTg}$-DI rats. Brain sections from 2-month-old $\mathrm{rTg}$ DI rats were immunolabeled with the mouse monoclonal antibody P2-1 to specifically detect human A $\beta P P$ (green) and the rabbit polyclonal antibody NeuN to detect neurons (red). A-F: There was widespread human A $\beta$ PP expression in neurons in the cortex (A and $\mathbf{B})$, hippocampus (C and $\mathbf{D})$, and thalamus ( $\mathbf{E}$ and $\mathbf{F}$ ). G: Immunoblot analysis of human A $\beta$ PP expression in total brain homogenates from wild-type (WT) and rTg-DI (Tg) rats. H: Quantitative immunoblotting was performed to measure total A $\beta P P$ (endogenous rat $\mathrm{A} \beta \mathrm{PP}+$ transgenic human $\mathrm{A} \beta \mathrm{PP}$ ) in brain homogenates of wild-type and $\mathrm{rTg}-\mathrm{DI}$ rats. The level of transgene human A $\mathrm{PPP}$ expression is approximately $50 \%$ the amount of endogenous rat A $\beta P P$. I and $\mathbf{J}$ : The levels of soluble (I) and insoluble (J) A $\beta 40$ and $A \beta 42$ peptides in the forebrain of progressively aged rats were measured by enzyme-linked immunosorbent assay, as described in Materials and Methods. The data are of triplicate measurements. Data are expressed as means $\pm S D(\mathbf{H}-\mathbf{J}) . n=5$ rats per group $(\mathbf{H}) . n=5$ to $6 \mathrm{rTg}-\mathrm{DI}$ rats per group $(\mathbf{I}$ and $\mathbf{J})$. ${ }^{*} P<0.05$. Scale bars: $10 \mu \mathrm{m}(\mathbf{A}, \mathbf{C}$, and $\mathbf{E}) ; 50 \mu \mathrm{m}(\mathbf{B}, \mathbf{D}$, and $\mathbf{F})$.

increase in cerebral microvascular amyloid deposition, with nearly $45 \%$ and $70 \%$ of capillaries covered in fibrillar amyloid in the thalamic and hippocampal regions, respectively (Figure 2J). Although the levels of capillary CAA were somewhat lower in the cortical areas, nearly $30 \%$ of the 

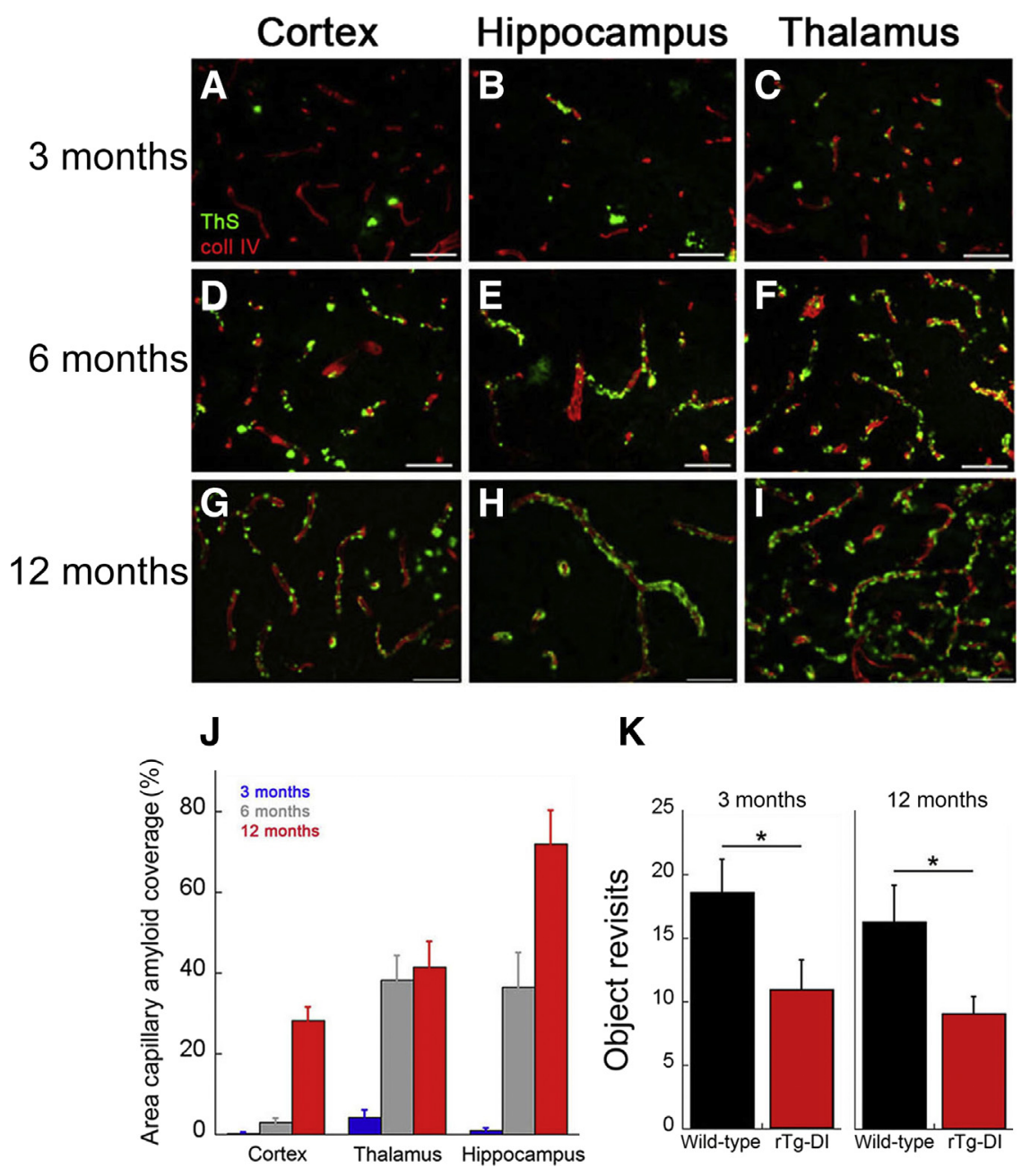

Figure 2 Progressive accumulation of microvascular cerebral amyloid angiopathy in $\mathrm{rTg}-\mathrm{DI}$ rats. A-I: Brain sections from rTg-DI rats at 3 months $(\mathbf{A}-\mathbf{C}), 6$ months $(\mathbf{D}-\mathbf{F})$, and 12 months $(\mathbf{G}-\mathbf{I})$ of age were stained for fibrillar amyloid using thioflavin $\mathrm{S}$ (green) and immunolabeled for collagen type IV (coll IV) to identify cerebral microvessels (red). The rTg-DI rats develop earlyonset and progressive cerebral microvascular fibrillar amyloid in the cortical, hippocampal, and thalamic regions. J: Quantitation of microvascular thioflavin S-positive amyloid load in different brain regions of 3-month-old (blue bars), 6month-old (gray bars), and 12-month-old (red bars) rTg-DI rats. K: rTg-DI rats show consistently fewer return approaches within a session to four novel objects placed in an open field arena compared with wild-type rats at 3 and 12 months of age. Data are expressed as means \pm SD. $n=6$ to $7 \mathrm{rTg}$-DI rats per group ( $\mathbf{J}$ and $\mathbf{K}$ ). ${ }^{*} P<0.05$. Scale bars $=50 \mu \mathrm{m}(\mathbf{A}-\mathbf{I})$.

vessel surface was covered. More important, these early levels of capillary CAA in the rTg-DI rats were associated with the characteristic slowing in the manner of exploration of unique objects arrayed in an open field (ie, the novel object exploration task), consistent with a form of perceptual slowing likely to result from corticothalamic and hippocampal dysfunction and present in $\mathrm{CAA}^{7}$ (Figure $2 \mathrm{~K}$ ).

Immunohistochemical analysis revealed that most cerebral microvascular amyloid was composed of $A \beta 40$ (Figure 3, A-C). Furthermore, ELISA analysis of isolated cerebral microvessels from rTg-DI rats confirmed that nearly $90 \%$ of the accumulated amyloid was composed of A 340 peptide (Figure 3, D and E). Fibrillar microvascular amyloid seeds were isolated from rTg-DI rats (Figure $3 \mathrm{~F}$ ), and FTIR spectroscopy revealed a strong $\beta$-sheet secondary structural component characteristic of amyloid fibrils, as indicated by the prominent spectral peak at approximately $1632 / \mathrm{cm}$ (Figure 3G). Although wild-type A $\beta 40$ peptide slowly formed fibrils, the addition of the isolated microvascular amyloid seeds promoted rapid fibril assembly of wild-type $A \beta 40$, as assayed by thioflavin $\mathrm{T}$ fluorescence spectroscopy (Figure $3 \mathrm{H}$ ). The rapid increase in fluorescence argues that the $\mathrm{A} \beta$ monomers are using the structural template provided by the seeds as they associate with the growing fibrils. Fibrils seeded from cerebral vascular amyloid exhibit intense FTIR bands characteristic of antiparallel $\beta$-strand structure. ${ }^{33}$ Notably, the newly formed seeded wild-type A $\beta 40$ fibrils from rTg-DI microvascular amyloid exhibit a similar antiparallel signature, as reflected by the strong FTIR spectral band at approximately 1607/cm (Figure 3I).

Accumulation of Cerebral Microvascular Amyloid in rTg-DI Rats Promotes Capillary Structural Changes and Perivascular Inflammation

The prominent accumulation of cerebral microvascular amyloid in rTg-DI rats (Figure 4B) caused pronounced structural changes to cerebral capillaries compared with similarly aged wild-type rats (Figure 4A). These capillary changes included the appearance of numerous string vessels, which are remnants of capillaries with no endothelial cells, ${ }^{46}$ and prominent vessel fragmentation (Figure 4B). These structural changes were accompanied by a significant 

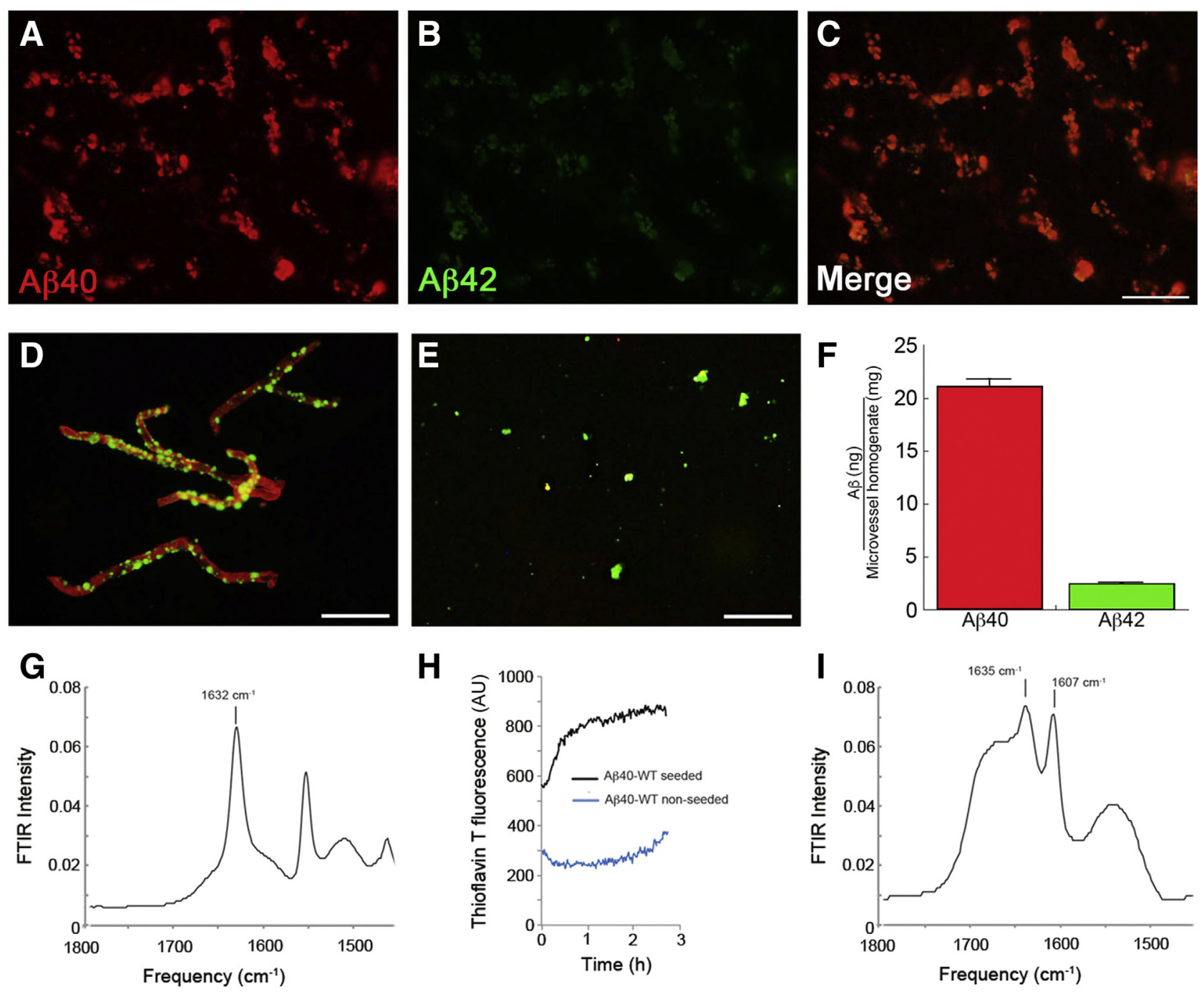

Figure 3 Analysis of cerebral microvascular $A \beta$ deposition in rTg-DI rats. A-C: Brain sections from rTg-DI rats at 12 months of age were immunolabeled with an antibody specific for $A \beta 40$ (red; A) and an antibody specific for A 442 (green; B) and merged (C). D: Cerebral microvessels were isolated from 12-month-old rTg-DI rats, stained with thioflavin $\mathrm{S}$ to visualize fibrillar amyloid deposits (green), and immunolabeled with an antibody to collagen IV to view the cerebral microvessels (red). E: Cerebral microvascular amyloid deposits after digestion and removal of the capillaries and stained for fibrillar amyloid using thioflavin S (green). F: Enzyme-linked immunosorbent assay measurements were performed to determine the amounts of $A \beta 40$ and $A \beta 42$ in the microvessels isolated from rTg-DI rat brain. G: Fourier transform infrared (FTIR) spectra in the region of the amide I vibration of vascular amyloid isolated from vessels of $\mathrm{rTg}$-DI rat brain. H: Thioflavin T fluorescence of fibril formation from seeds obtained from isolated vascular amyloid. Thioflavin fluorescence increases rapidly on the addition of $100 \mu \mathrm{mol} / \mathrm{L}$ A 40 -wild-type (WT) monomer to sonicated vascular amyloid (black). Monomeric Aß40-WT peptide exhibits a much slower increase in fluorescence in the absence of fibril seeds (blue). I: FTIR spectra in the region of the amide I vibration of A $\beta 40$-WT fibrils produced from seeds of the rat vascular amyloid. The amide I vibration at $1632 / \mathrm{cm}$ corresponds to $\beta$-sheet. The vibration at $1607 / \mathrm{cm}$ corresponds to the amide vibration shifted as a result of ${ }^{13} \mathrm{C}=0$ labels at the backbone carbonyls of Leu17, Ala21, Gly33, and Gly37. The large intensity of the $1607 / \mathrm{cm}$ band relative to the $\beta$-sheet vibration at $1632 / \mathrm{cm}$ is characteristic of antiparallel $\beta$-sheet structure. The spectra were obtained after incubation for 48 hours at $37^{\circ} \mathrm{C}$. Data are expressed as means $\pm \mathrm{SD}(\mathbf{F}) . n=5 \mathrm{rTg}$-DI rats $(\mathbf{F})$. Scale bars $=50 \mu \mathrm{m}(\mathbf{A}-\mathbf{E})$. AU, arbitrary unit.

$(P<0.001)$ increase in the tortuosity of the cerebral capillaries in the affected brain regions, further demonstrating the deleterious effects of vascular amyloid accumulation (Figure 4C). In addition, there was a significant $(P<0.001)$ increase in capillary area in the thalamic region and an increased trend in the other brain regions that may reflect the increased tortuosity (Figure 4D).

The cerebral microvascular amyloid in rTg-DI rats, which engages the surrounding brain parenchyma, was also found to be associated with a strong neuroinflammatory response reflected by a robust increase of perivascular reactive astrocytes (Figure 5B) when compared with similarly aged wild-type rats devoid of microvascular amyloid (Figure 5A). Likewise, an elevated number of perivascular activated microglia are present in rTg-DI rats in areas with microvascular CAA (Figure 5D). The lower number of microglia present in the similarly aged wild-type rats were in the resting ramified surveillance mode with numerous extended processes (Figure 5C), whereas the elevated numbers of microglia in rTg-DI rats exhibited a bushy, activated 

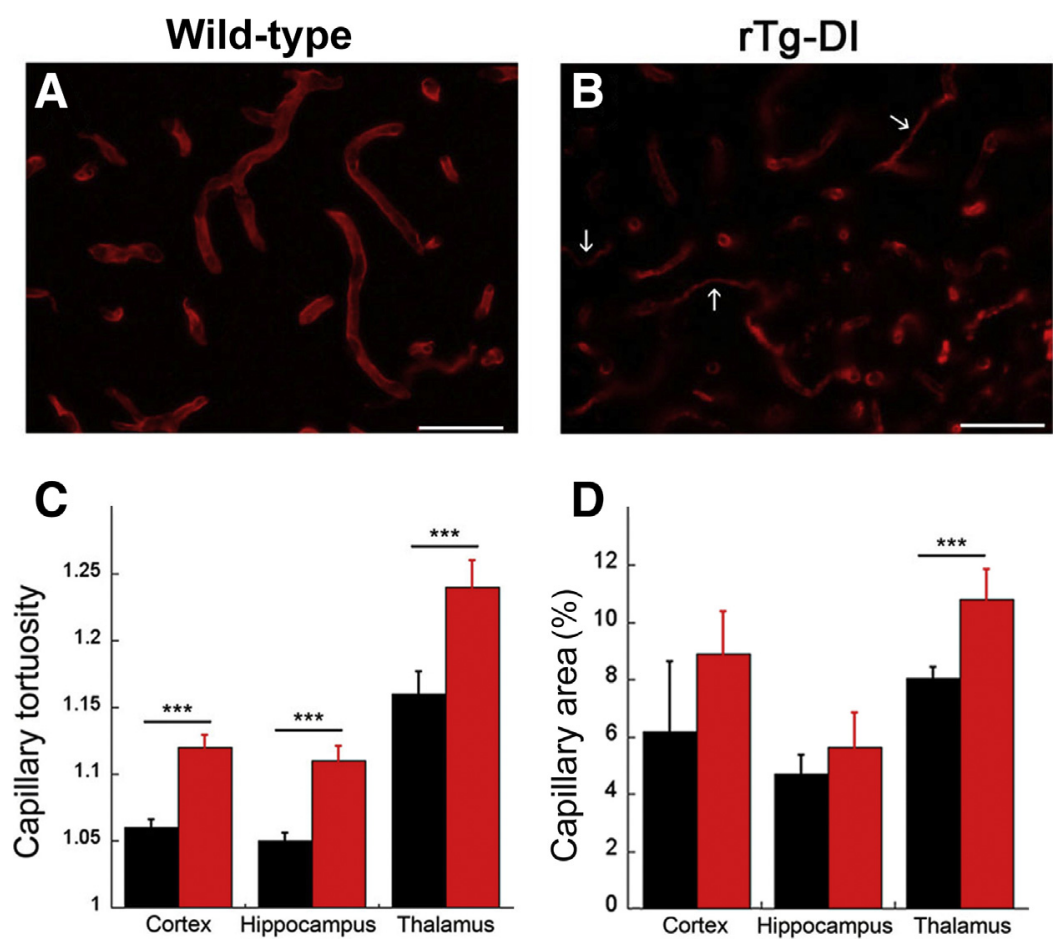

Figure 4 Cerebral microvascular structural changes in rTg-DI rats. $\mathbf{A}$ and $\mathbf{B}$ : Brain sections from wild-type $(\mathbf{A})$ and rTg-DI (B) rats at 12 months of age were immunolabeled with an antibody to collagen IV to identify cerebral microvessels. Images shown are from the thalamic region of the rats. Numerous fragmented and string vessels (arrows) were identified in the rTg-DI rats. C: The tortuosity of the cerebral capillaries was measured in the cortex, hippocampus, and thalamic region of wild-type and $\mathrm{rTg}$-DI rats. D: The capillary area was measured in the cortex, hippocampus, and thalamic region of wild-type and $\mathrm{rTg}$-DI rats. The data show that cerebral capillaries in rTg-DI rats exhibit structural abnormalities, increased tortuosity, and, in the thalamic region, increased vascularization. Data are expressed as means \pm SD. $n=5$ to 6 rats per group (C and $\mathbf{D}) .{ }^{* *} P<0.001$. Scale bars $=50 \mu \mathrm{m}(\mathbf{A}$ and $\mathbf{B})$. morphology actively engaged with the fibrillar amyloid deposits on the cerebral capillaries (Figure 5D). These findings indicate that the presence of prominent cerebral microvascular amyloid in rTg-DI rats is accompanied by structural deterioration of brain capillaries and a robust increase in activated perivascular glial cells.

\section{Robust Microhemorrhages and Small-Vessel Occlusions Are Detected by MRI in rTg-DI Rats}

A prominent clinical feature of CAA in humans is the presence of cerebral microbleeds. ${ }^{1,2}$ The presence of microbleeds was histochemically evaluated by Perl's staining for perivascular hemosiderin deposits in groups of progressively aged rTg-DI rats. Evidence of cerebral microbleeds was observed in the cortical and hippocampal regions of 12-month-old rTg-DI rats (Figure 6, A and B). However, microbleeds were particularly abundant in the thalamic region of the brain, with various presentations of perivascular iron deposits, including tightly associated crescent-shaped deposits (Figure 6C), complete circumferential involvement (Figure 6D), indications of multiple bleeds in several adjacent microvessels (Figure 6E), and more diffuse perivascular iron deposits (Figure 6F). Because it was often difficult to discriminate between individual and grouped microbleeds, the area of iron deposition in the different brain regions was measured at 6 and 12 months. Signs of microbleeds in the cortex were not observed at 6 months and were low at 12 months (Figure 6G). However, in the hippocampus, small areas of microbleeds were detected at 6 months, and this increased as the rTg-DI rats aged to 12 months. Most notably, in the thalamus, there was clear evidence of microbleeds at 6 months, which dramatically increased at 12 months of age with $>1 \%$ of the total thalamic area covered with perivascular iron deposits. Although microbleeds are numerous in 12-month-old rTg-DI rats, large macrobleeds have not been observed at this age.

Small-vessel occlusions and calcifications are other microvasculopathies observed in CAA patients. ${ }^{2,47,48}$ Interestingly, in the thalamic region of prevalent microbleeds in rTg-DI rats, numerous occluded microvessels were observed (Figure 7A). Calcium staining of adjacent brain tissue sections indicated calcifications in these small-vessel occlusions surrounded by areas of fibrinoid necrosis (Figure 7B). These microvascular occlusions were bilateral and largely restricted to the ventral posterior lateral nucleus of the thalamic region (Figure $7 \mathrm{C}$ ). The overall numbers of these thalamic small-vessel occlusions were quantified in rTg-DI rats as they aged from 3 to 12 months. The occlusions were extremely rare or absent at 3 months of age (Figure 7D). However, at 6 and 12 months, the numbers markedly increased to approximately 40 and approximately 115 small-vessel occlusions, respectively, in this thalamic region. At each age, most vessel occlusions were in capillaries, but occasionally larger microvessels were also occluded.

Studies were performed in rTg-DI rats to characterize the microhemorrhages in situ using in vitro MR microscopy. Figure 8 shows the results of MRI-to-histology matching from a 12-month-old rTg-DI rat with documented thalamic 


\section{Wild-type}
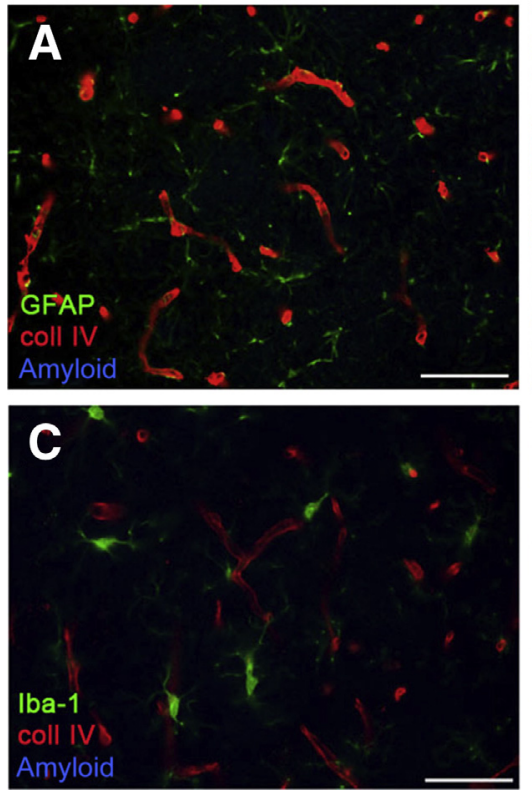

\section{rTg-DI}
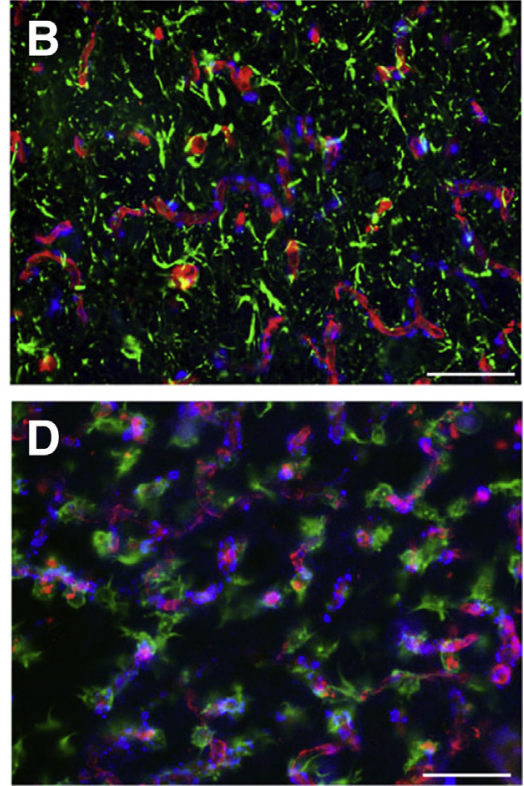

Figure 5 Perivascular glial activation in rTg-DI rats. Twelve-month-old brain sections from wild-type (A and C) and rTg-DI (B and D) rats were stained for fibrillar amyloid (blue), immunolabeled with an antibody to collagen IV (coll IV) to identify cerebral microvessels (red), and immunolabeled with an antibody to glial fibrillary acidic protein (GFAP) to identify astrocytes (green; A and $\mathbf{B}$ ) or immunolabeled with an antibody to Iba-1 to identify microglia (green; $\mathbf{C}$ and $\mathbf{D}$ ). $\mathbf{r T g}$-DI rats exhibit strong increases in perivascular reactive astrocytes and activated microglia in response to the prominent cerebral microvascular amyloid. Scale bars $=50 \mu \mathrm{m}$ (A-D). microhemorrhages and small-vessel occlusions. A twodimensional T2 $*$-weighted MRI (echo time $=7$ milliseconds) (Figure 8) was matched to the corresponding Perl iron-stained histologic slice (Figure 8). The dense dark areas in the thalamus shown on the T2*-weighted MRI are corresponding to the microvessels identified by Perl-stained iron deposits associated with the microbleeds and occluded vessels. The Perl-stained histologic slice is overlaid on the $\mathrm{T} 2 *$-weighted images under conditions where the histologic slice has been rendered transparent to highlight the low signal intensity susceptibility features on the T2*-weighted MRI observed in the thalamus, bilaterally, which is caused by the presence of iron. Together, these findings indicate that rTg-DI rats develop numerous and consistent thalamic microhemorrhages and microvessel occlusions that are readily detected by $\mathrm{T} 2 *$-weighted MRI.

\section{Discussion}

CAA is a common cerebral small-vessel disease of elderly individuals and a prominent comorbidity of $\mathrm{AD}$ that promotes and exacerbates VCID, yet our understanding of the condition remains limited; despite the high prevalence, there is no effective treatment. Several transgenic mouse models, which develop varying levels of CAA, have been in use for more than a decade and have provided better insights into the pathogenesis of CAA. ${ }^{23-32}$ However, their usefulness to
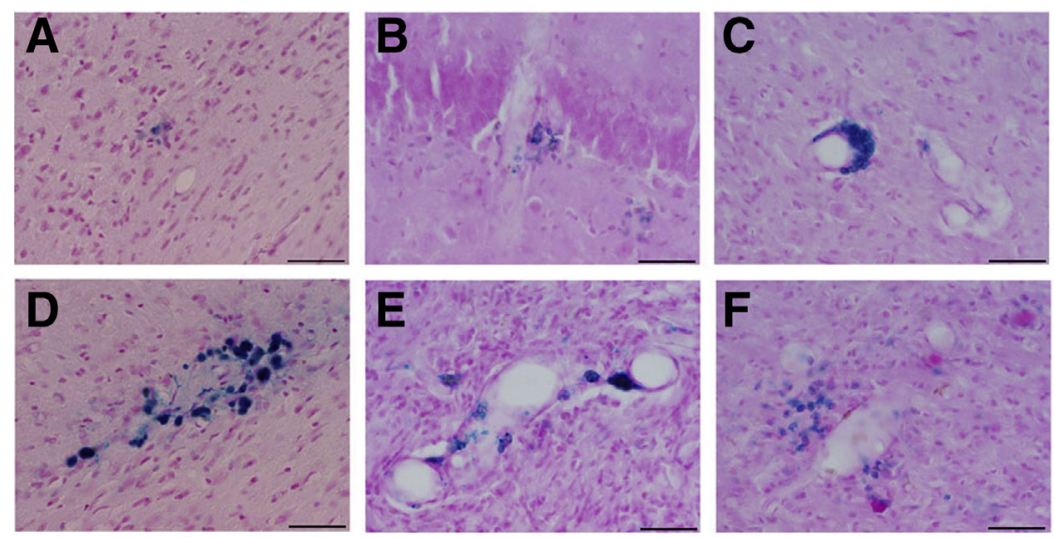
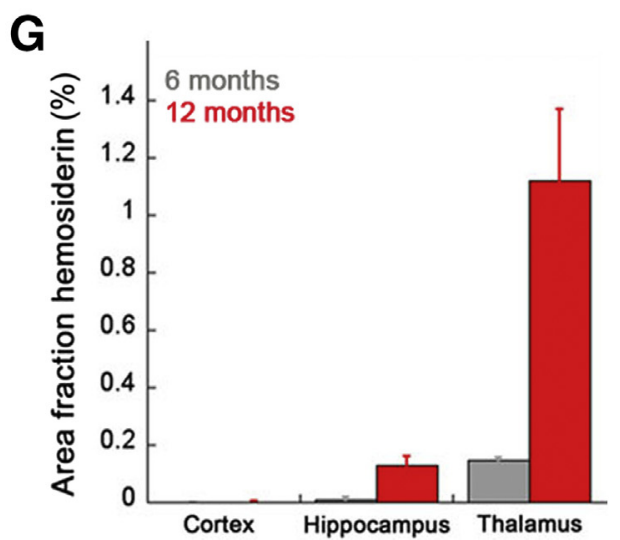

Figure 6 Prominent cerebral microhemorrhages in rTg-DI rats. A-F: Twelve-month-old brain sections from rTg-DI rats were stained for hemosiderin to identify microhemorrhages (blue) in the cortex (A), hippocampus (B), and the thalamus (C-F). G: The percentage area fraction of hemosiderin staining was quantitated in the cortex, hippocampus, and thalamus of 6-month-old (gray) and 12-month-old (red) rTg-DI rats. Progressive and consistent accumulation of microbleeds was observed, particularly in the thalamus, of rTg-DI rats. Data are expressed as means \pm SD. $n=6$ to $7 \mathrm{rTg}$-DI rats per group (G). Scale bars $=50 \mu \mathrm{m}(\mathbf{A}-\mathbf{F})$. 

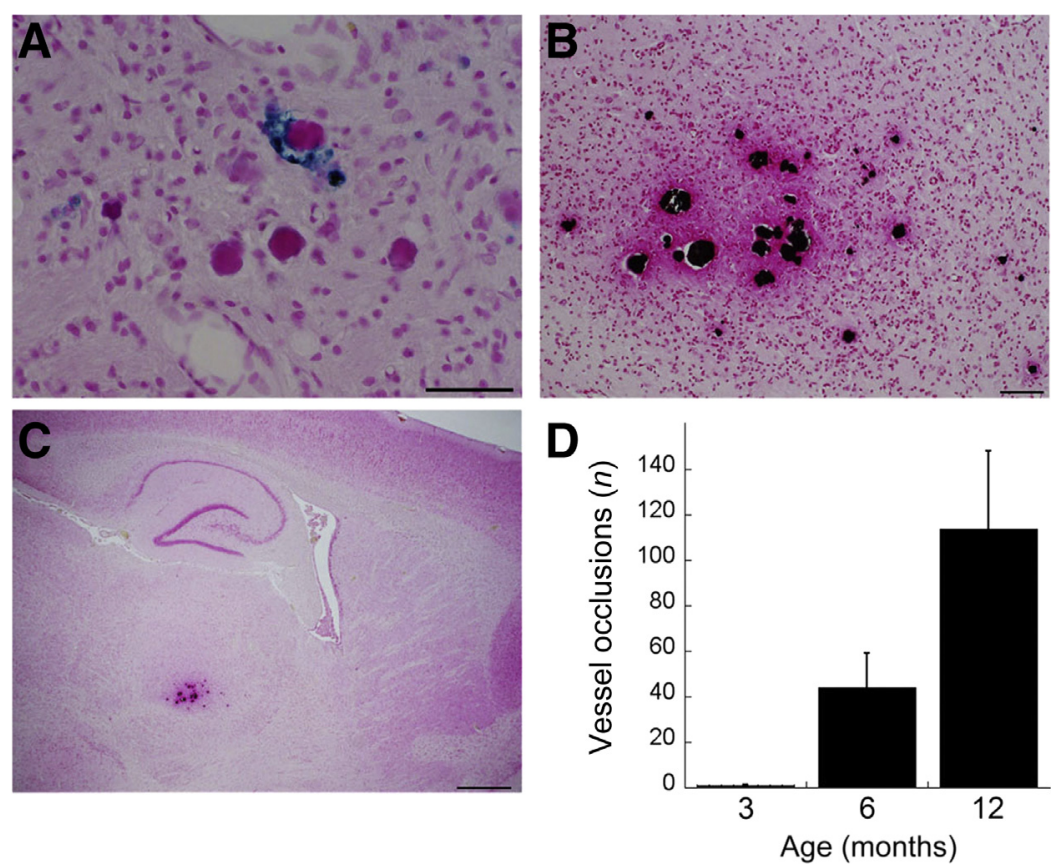

Figure 7 Progressive cerebral microvascular occlusions in rTg-DI rats. A: Twelve-month-old brain sections from $\mathrm{rTg}$-DI rats were stained for hemosiderin to identify microhemorrhages (blue) and counterstained with pararosaniline (pink) in the thalamus. B: Adjacent tissue sections were stained for calcium (black) and counterstained with pararosaniline (pink) in the thalamus. C: Lower magnification revealed that the occluded, calcified microvessels were restricted to the thalamic region. D: The number of thalamic microvessel occlusions was counted in progressively aged rTg-DI rats. Numerous occluded capillaries/microvessels were observed around microbleeds specifically in the thalamic region of $\mathrm{rTg}$-DI rats. Data are expressed as means \pm SD. $n=5$ to $6 \mathrm{rTg}$-DI rats per group (D). Scale bars: $50 \mu \mathrm{m}$ (A and B); $200 \mu \mathrm{m}$ (C). further investigate CAA from the point of view of faithfully recapitulating the human condition is limited because of their biological evolutionary distance from humans and the small size of their brains, which can be problematic for effective neuroimaging in vivo. Furthermore, complicated cognitive testing to investigate VCID in the setting of evolving CAA can be unreliable because of temperamental variability in mice. ${ }^{49}$ Although naturally occurring models of CAA exist, most notably in canines and nonhuman primates, the experimental use of these species for large cohort experimentation and testing is limited because of the cost and time needed for CAA to develop (decade or decades). ${ }^{50}$ Thus, there is a need for a better experimental model of CAA. The use of rats, specifically transgenic rats, to investigate the pathogenesis of human central nervous system disorders offers several distinct advantages over transgenic mice. First, rats are evolutionarily 4 to 5 million years closer to humans. ${ }^{51}$ Thus, the genetics, physiology, and brain morphology of rats are better suited to model human CAA. Second, the larger brain size and increased white/gray matter ratio of the rat enables structural neuroimaging studies with superior contrast/noise and signal/noise ratios compared with mice. Last, rats provide the opportunity for far more sophisticated cognitive testing compared with mice, thereby bridging a gap in knowledge as to how CAA drives VCID. ${ }^{52}$

Herein, we describe the generation and initial characterization of a novel transgenic rat model (rTg-DI) of microvascular type $1 \mathrm{CAA}$. The rTg-DI rats were designed from our earlier success in generating Tg-SwDI transgenic mice that develop early-onset and progressive cerebral microvascular amyloid deposition. ${ }^{27}$ rTg-DI rats express the human A $\beta P P$ transgene harboring the Swedish familial AD mutations, the $\beta$-secretase cleavage site, and the chimeric Dutch E693Q/Iowa D694N familial CAA mutations in neurons in the brain. Indeed, low-level neuronal expression of the human A $\beta P P$ transgene was observed in the rTg-DI rats (Figure 1). This phenotype is important because it signifies that the new CAA model does not require abnormal, vast overexpression of human $\mathrm{A} \beta \mathrm{PP}$ and production of human $\mathrm{A} \beta$, which is commonly found in other transgenic rodent models and can introduce potential confounds on neuronal function. This underscores that the robust cerebral accumulation of $\mathrm{A} \beta$ and the specific development of microvascular CAA are a consequence of the chimeric familial Dutch/Iowa mutations in the $A \beta$ peptide that is produced in the brains of these rats. Interestingly, the earlyonset development of microvascular CAA and associated neuroinflammation coincide with early-onset and sustained cognitive deficits in rTg-DI rats and offer the promise of a unique model for the future elucidation as to how CAA can promote VCID.

The type and form of amyloid that accumulates in the cerebral microvasculature of rTg-DI rats is remarkably similar to what is found in human CAA. For example, in contrast to parenchymal amyloid plaques that largely accumulate fibrillar $A \beta 42$ in both humans and rodent models, the fibrillar cerebral vascular amyloid deposits in rTg-DI rats are composed primarily of the shorter $A \beta 40$ species. ${ }^{53,54}$ In addition to the difference in the length of the $A \beta$ peptide that forms the amyloid fibrils in either parenchymal plaques or cerebral vascular deposits, other microstructural differences have been found. Fibrils produced from seeds of cerebral vascular fibrillar amyloid deposits exhibit increased intensity 

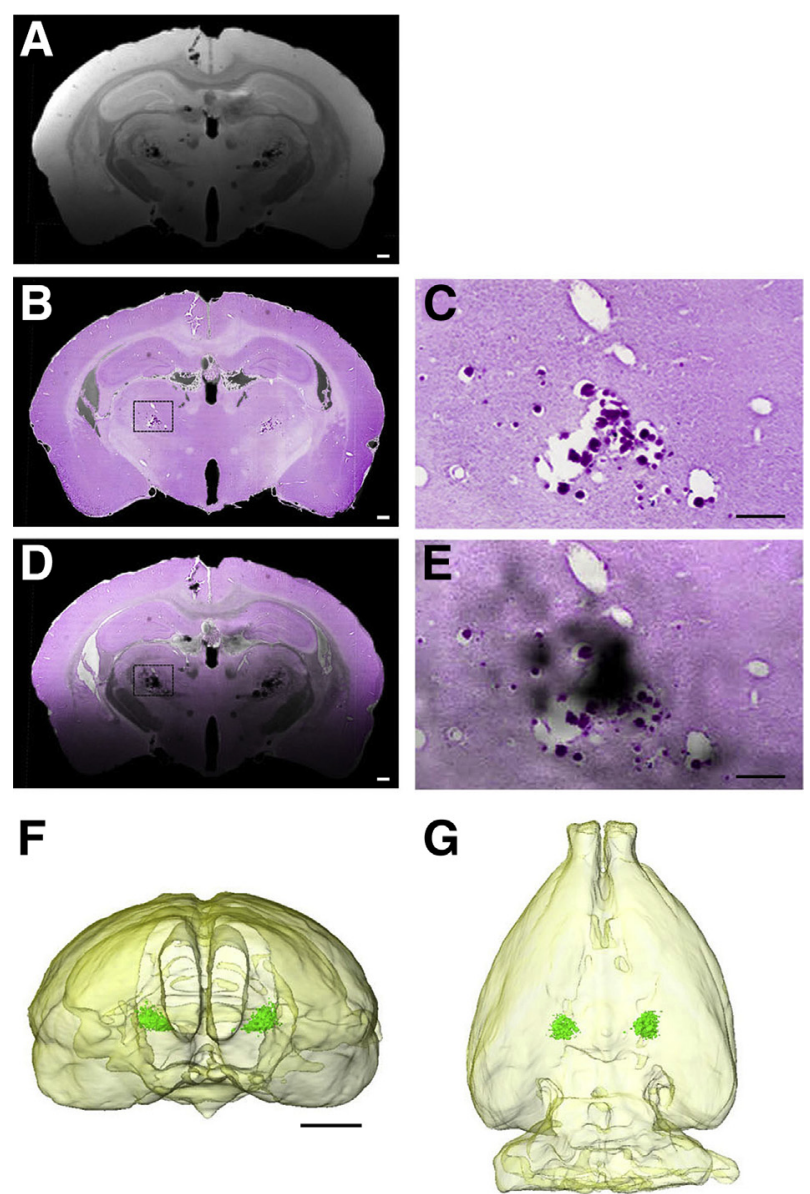

Figure 8 Detection of microhemorrhage by magnetic resonance imaging (MRI) in rTg-DI rats. A and B: Two-dimensional T2*-weighted MRI (echo time $=7$ milliseconds; $\mathbf{A}$ ) matched to the corresponding Perl-stained histologic slice (B). A: The dense dark areas in the thalamus shown on the T2*-weighted MRI correspond to microvessels characterized by Perl stain-positive microbleeds and occluded vessels. D: Perl-stained histologic slice was overlaid on the $\mathrm{T}^{*}$-weighted images under conditions where the histologic slice has been rendered transparent to highlight the low signal intensity susceptibility features on the $\mathrm{T} 2^{*}$-weighted MRI observed bilaterally in the thalamus, which is caused by presence of hemosiderin. $\mathbf{C}$ and E: Higher magnifications of the boxed areas in $\mathbf{B}$ and $\mathbf{D}$, respectively. $\mathbf{F}$ and G: Three-dimensional volume-rendered MRIs of the same rat showing the brain outlined in light yellow; and the Perl stain-positive associated low signal intensity area in the thalamus has been volume rendered (green) to illustrate the near-perfect symmetry of the microhemorrhage/occluded microvessel areas. Scale bars: $100 \mu \mathrm{m}$ (C and E); $500 \mu \mathrm{m}$ (A, B, and D); 3.5 $\mathrm{mm}$ ( $\mathbf{F}$ and $\mathbf{G})$.

in FTIR spectra that is characteristic of antiparallel structure of the $\beta$ strands forming the backbone of the $A \beta$ fibrils. ${ }^{33}$ This structure is distinct from the parallel registry observed in parenchymal plaque amyloid fibrils. ${ }^{55,56}$ The $\beta$-sheet, antiparallel fibril character is also observed in the cerebral microvascular amyloid deposits of the rTg-DI rats (Figure 3). This important distinction between parenchymal and cerebral vascular amyloid fibrils likely is involved in the pathogenesis of these two types of deposits and may have important consequences with regard to subsequent pathologies associated with each lesion type.
The accumulation of cerebral microvascular amyloid in rTg-DI rats promotes distinct vessel and perivascular changes that are reflected in human CAA. Structurally, the accumulation of microvascular amyloid causes significant alterations in capillary morphology, including increased vessel fragmentation, occlusion, and tortuosity (Figure 4). It has been suggested that these types of structural alterations can impair capillary blood flow and may cause hypertension upstream from these sites of disruption. ${ }^{57}$ On the other hand, capillary tortuosity caused by microvascular amyloid can lead to poor perfusion and downstream infarction. ${ }^{22,58}$ Interestingly, there was an increase in the capillary area in the thalamic region of rTg-DI rats that might reflect the measured increase in capillary tortuosity. Alternatively, it is possible that there is an increase in vascularization in response to the extensive amyloid-mediated damage to the microvasculature in this region. The changes in capillary morphology likely contribute to increased perivascular disturbances in fluid homeostasis, inflammation, thrombosis, and microhemorrhage observed in CAA. In line with this, the accumulation of fibrillar amyloid along the capillaries of rTg-DI rats induces a strong neuroinflammatory response characterized by activation and robust increases in perivascular astrocytes and microglia, which is accompanied by consistent and frequent microbleeds and microvascular occlusions, particularly in the thalamic region. These latter thrombotic events may result directly from the excessive amyloid deposition in this region but are more likely to be related to downstream inflammatory responses triggered by amyloid accumulation.

Currently, outside of biopsy or autopsy confirmation, the clinical diagnosis of CAA is based on the Boston criteria, which relies primarily on the detection of lobar microbleeds on the basis of MR imaging hypointensities resulting from perivascular hemosiderin. ${ }^{3,59}$ Similarly, MR imaging analysis of rTg-DI rats readily detected the perivascular hemosiderin associated with the prevalent thalamic microbleeds. $3 \mathrm{D}$ reconstruction of the MR-defined hypointensities illustrated the symmetrical and bilateral nature of these consistent thalamic microbleeds (Figure 8). These findings suggest that similar parameters for the Boston criteria diagnosis of CAA in humans can be applied to rTg-DI rats, providing opportunity to investigate the longitudinal development and persistence of these hallmark lesions by clinically relevant methods.

Although the extent of CAA, capillary structural changes, and perivascular neuroinflammation is high in several brain regions of rTg-DI rats at 6 to 12 months, it is currently unclear why the most severe associated events of microhemorrhage and vessel occlusion are largely restricted bilaterally to the thalamic regions. In human CAA, cerebral microbleeds tend to be found in lobar cortical regions, in particular the occipital cortex, and not so frequently in deeper brain structures. However, in cerebral autosomal dominant arteriopathy with subcortical infarcts and leukoencephalopathy, another type of cerebral small-vessel 
disease, thalamic microbleeds are commonly observed in both humans and the equivalent transgenic mouse model. ${ }^{60-62}$ Perhaps the thalamic region in rats exhibits differential blood flow or perivascular clearance mechanisms compared with humans and in other regions of the rat brain, making this region particularly susceptible to excessive perivascular amyloid deposition. Alternatively, rat capillaries/microvessels in the thalamic areas may possess a different vascular environment compared with other brain regions. Future studies are needed to evaluate these possibilities. Although the anatomic distribution of CAA and cerebral vascular thrombotic lesions may be somewhat different between rTg-DI rats and humans, this unique model provides new opportunities to investigate the pathogenic development of CAA and its relation to VCID. Furthermore, rTg-DI rats provide an improved platform for the development of biomarkers and preclinical testing of therapeutic interventions for this common small-vessel disease.

\section{Acknowledgment}

Antibody reagents for the amyloid $\beta$ enzyme-linked immunosorbent assay were kindly provided by Lilly Research Laboratories (Indianapolis, IN).

\section{References}

1. Banerjee G, Carare R, Cordonnier C, Greenberg SM, Schneider JA, Smith EE, Buchem MV, Grond JV, Verbeek MM, Werring DJ: The increasing impact of cerebral amyloid angiopathy: essential new insights for clinical practice. J Neurol Neurosurg Psychiatry 2017, 88: 982-994

2. Auriel E, Greenberg SM: The pathophysiology and clinical presentation of cerebral amyloid angiopathy. Curr Atheroscler Rep 2012, $14: 343-350$

3. Biffi A, Greenberg SM: Cerebral amyloid angiopathy: a systematic review. J Clin Neurol 2011, 7:1-9

4. Greenberg SM, Gurol ME, Rosand J, Smith EE: Amyloid angiopathy-related vascular cognitive impairment. Stroke 2004, 35: $2616-2619$

5. Attems J, Jellinger K, Thal DR, Van Nostrand W: Review: sporadic cerebral amyloid angiopathy. Neuropathol Appl Neurobiol 2011, 37: 75-93

6. Thal DR, Ghebremedhin E, Rub U, Yamaguchi H, Del Tredici K, Braak H: Two types of sporadic cerebral amyloid angiopathy. J Neuropathol Exp Neurol 2002, 61:282-293

7. Arvanitakis Z, Leurgans SE, Wang Z, Wilson RS, Bennett DA, Schneider J: Cerebral amyloid angiopathy pathology and cognitive domains in older persons. Ann Neurol 2011, 69:320-327

8. Boyle PA, Yu L, Nag S, Leurgans S, Wilson RS, Bennett DA, Schneider J: Cerebral amyloid angiopathy and cognitive outcomes in community-based older persons. Neurology 2015, 85:1930-1936

9. Jellinger KA: Alzheimer disease and cerebrovascular pathology: an update. J Neural Transm 2002, 109:813-836

10. Launer LJ, Petrovitch H, Ross GW, Markesbery W, White LR: AD brain pathology: vascular origins? results from the HAAS autopsy study. Neurobiol Aging 2008, 29:1587-1590

11. Esiri M, Chance S, Joachim C, Warden D, Smallwood A, Sloan C, Christie S, Wilcock G, Smith AD: Cerebral amyloid angiopathy, subcortical white matter disease and dementia: literature review and study in OPTIMA. Brain Pathol 2015, 25:51-62

12. Levy E, Carman M, Fernandez-Madrid IJ, Power MD, Lieberburg I, van Duinen S, Bots GT, Luyendijk W, Frangione B: Mutation of the Alzheimer's disease amyloid gene in hereditary cerebral hemorrhage, Dutch type. Science 1990, 248:1124-1126

13. Van Broeckhoven C, Haan J, Bakker E, Hardy JA, Van Hul W, Wehnert A, Vegter-Van der Vlis M, Roos RA: Amyloid $\beta$ protein precursor gene and hereditary cerebral hemorrhage with amyloidosis (Dutch). Science 1990, 248:1120-1122

14. Grabowski TJ, Cho HS, Vonsattel JP, Rebeck GW, Greenberg SM: Novel amyloid precursor protein mutation in an Iowa family with dementia and severe cerebral amyloid angiopathy. Ann Neurol 2001, 49:697-705

15. Rensink AA, de Waal RM, Kremer B, Verbeek MM: Pathogenesis of cerebral amyloid angiopathy. Brain Res Brain Res Rev 2003, 43: 207-223

16. Eikelenboom P, Veerhuis R, Familian A, Hoozemans JJ, van Gool WA, Rozemuller AJM: Neuroinflammation in plaques and vascular $\beta$-amyloid disorders: clinical and therapeutic implications. Neurodegener Dis 2008, 5:190-193

17. Richard E, Carrano A, Hoozemans JJ, van Horssen J, van Haastert ES, Eurelings LS, de Vries HE, Thal DR, Eikelenboom P, van Gool WA, Rozemuller AJ: Characteristics of dyshoric capillary cerebral amyloid angiopathy. J Neuropathol Exp Neurol 2010, 69:1158-1167

18. Thal DR, Ghebremedhin E, Orantes M, Wiestler OD: Vascular pathology in Alzheimer's disease: correlation of cerebral amyloid angiopathy and arteriosclerosis/lipohyalinosis with cognitive decline. J Neuropathol Exp Neurol 2003, 62:1287-1301

19. Attems J, Jellinger KA: Only cerebral capillary amyloid angiopathy correlates with Alzheimer pathology: a pilot study. Acta Neuropathol 2004, 107:83-90

20. Bailey TL, Rivara CB, Rocher AB, Hof PR: The nature and effects of cortical microvascular pathology in aging and Alzheimer's disease. Neurol Res 2004, 26:573-578

21. Rozemuller AJ, van Gool WA, Eikelenboom P: The neuroinflammatory response in plaques and amyloid angiopathy in $\mathrm{Alz}-$ heimer's disease: therapeutic implications. Curr Drug Targets CNS Neurol Disord 2005, 4:223-233

22. Hecht M, Kramer LM, von Arnim CAF, Otto M, Thal DR: Capillary cerebral amyloid angiopathy in Alzheimer's disease: association with allocortical/hippocampal microinfarcts and cognitive decline. Acta Neuropathol 2018, 135:681-694

23. Games D, Adams D, Alessandrini R, Barbour R, Berthelette P, Blackwell C, Carr T, Clemens J, Donaldson T, Gillespie F, Guido T, Hagopian S, Johnson-Wood K, Khan K, Lee M, Leibowitz P, Lieberberg I, Little S, Masliah E, McConlogue L, MontoyaZavala M, Mucke L, Paganini L, Penniman E, Power M, Schenk D, Seubert P, Snyder B, Soriano F, Tan H, Vitale J, Wadsworth S, Wolozin B, Zhao J: Alzheimer-type neuropathology in transgenic mice overexpressing V717F $\beta$-amyloid precursor protein. Nature 1995, 373:523-527

24. Hsiao K, Chapman P, Nilsen S, Eckman C, Harigaya Y, Younkin S, Yang F, Cole G: Correlative memory deficits, $A \beta$ elevation, and amyloid plaques in transgenic mice. Science 1996, 274:99-102

25. Calhoun ME, Burgermeister P, Phinney AL, Stalder M, Tolnay M, Wiederhold K-H, Abramowski D, Sturchler-Pierrat C, Sommer B, Staufenbiel M, Jucker M: Neuronal overexpression of mutant amyloid precursor protein results in prominent deposition of cerebrovascular amyloid. Proc Natl Acad Sci U S A 1999, 96: 14088-14093

26. Oakley H, Cole SL, Logan S, Maus E, Shao P, Craft J, GuillozetBongaarts A, Ohno M, Disterhoft J, Van Eldik L, Berry R, Vassar R: Intraneuronal $B$-amyloid aggregates, neurodegeneration, and neuron loss in transgenic mice with five familial Alzheimer's disease mutations: potential factors in amyloid plaque formation. J Neurosci 2006, 26:10129-10140 
27. Davis J, Xu F, Deane R, Romanov G, Previti ML, Zeigler K, Zlokovic BV, Van Nostrand WE: Early-onset and robust cerebral microvascular accumulation of amyloid $\beta$-protein in transgenic mice expressing low levels of a vasculotropic Dutch/Iowa mutant form of amyloid $\beta$-protein precursor. J Biol Chem 2004, 279:20296-20306

28. Herzig MC, Winkler DT, Burgermeister P, Pfeifer M, Kohler E, Schmidt SD, Danner S, Abramowski D, Sturchler-Pierrat C, Buki K, van Duinen SG, Maat-Schieman MLC, Staufenbiel M, Matthews PM, Jucker M: $A \beta$ is targeted to the vasculature in a mouse model of hereditary cerebral hemorrhage with amyloidosis. Nat Neurosci 2004, 7:954-960

29. Miao J, Xu F, Davis J, Otte-Holler I, Verbeek MM, Van Nostrand WE: Cerebral microvascular amyloid $\beta$ protein deposition induces vascular degeneration and neuroinflammation in transgenic mice expressing human vasculotropic mutant amyloid $\beta$ precursor protein. Am J Pathol 2005, 167:505-515

30. Miao J, Vitek MP, Xu F, Previti ML, Davis J, Van Nostrand WE: Reducing cerebral microvascular amyloid $\beta$ protein deposition diminishes regional neuroinflammation in vasculotropic mutant amyloid precursor protein transgenic mice. J Neurosci 2005, 25:6271-6277

31. Xu F, Grande AM, Robinson JK, Previti ML, Davis J, Van Nostrand WE: Early-onset subicular microvascular amyloid and neuroinflammation correlate with behavioral deficits in vasculotropic mutant AßPP transgenic mice. Neuroscience 2007, 146:98-107

32. Xu W, Xu F, Anderson ME, Kotarba AE, Davis J, Robinson JK, Van Nostrand WE: Cerebral microvascular rather than parenchymal amyloid $\beta$-protein pathology promotes early cognitive impairment in transgenic mice. J Alzheimers Dis 2014, 38:621-632

33. Xu F, Fu Z, Dass S, Kotarba AE, Davis J, Smith SO, Van Nostrand WE: Cerebral vascular amyloid seeds drive amyloid $\beta$-protein fibril assembly with a distinct anti-parallel structure. Nat Commun 2016, 7:13527

34. Van Nostrand WE, Wagner SL, Suzuki M, Choi BH, Farrow JS, Geddes JW, Cotman CW, Cunningham DD: Protease nexin-II, a potent anti-chymotrypsin, shows identity to amyloid b-protein precursor. Nature 1989, 341:546-549

35. Johnson-Wood K, Lee M, Motter R, Hu K, Gordon G, Barbour R, Khan K, Gordon M, Tan H, Games D, Lieberburg I, Schenk D, Seubert P, McConlogue L: Amyloid precursor protein processing and A $\beta 42$ deposition in a transgenic mouse model of Alzheimer's disease. Proc Natl Acad Sci U S A 1997, 94:1550-1555

36. DeMattos RB, O'dell M, Parsadanian M, Taylor JW, Harmony JA, Bales KR, Paul SM, Aronow BJ, Holtzman DM: Clusterin promotes amyloid plaque formation and is critical for neuritic toxicity in a mouse model of Alzheimer's disease. Proc Natl Acad Sci U S A 2002, 99:10843-10848

37. Zlokovic BV, Mackic JB, Wang L, McComb JG, McDonough A: Differential expression of Na,K-ATPase alpha and beta subunit isoforms at the blood-brain barrier and the choroid plexus. J Biol Chem 1993, 268:8019-8025

38. Deane R, Yan SD, Submamaryan RK, LaRue B, Jovanovic S, Hogg E, Welch D, Manness L, Lin C, Yu J, Zhu H, Ghiso J, Frangione B, Stern A, Schmidt AM, Armstrong DL, Arnold B, Liliensiek B, Nawroth P, Hofman F, Kindy M, Stern D, Zlokovic B: RAGE mediates amyloid beta-peptide transport across the bloodbrain barrier and accumulation in brain. Nat Med 2003, 9:907-913

39. Gomori G: Microtechnical demonstration of iron. Am J Pathol 1936, 12:655-663

40. Winkler DT, Bondolfi L, Herzig MC, Jann L, Calhoun ME, Weiderhold KH, Tolnay M, Staufenbiel M, Jucker M: Spontaneous hemorrhagic stroke in a mouse model of cerebral amyloid angiopathy. J Neurosci 2001, 21:1619-1627

41. Rungby J, Kassem M, Fink Eriksen E, Danscher G: The von Kossa reaction for calcium deposits: silver lactate staining increases sensitivity and reduces background. Histochem J 1993, 25:446-451

42. Long JM, Kalehua AN, Muth NJ, Hengemihle JM, Jucker M, Calhoun ME, Ingram DK, Mouton PR: Stereological estimation of total microglia number in mouse hippocampus. J Neurosci Methods 1998, 84: $101-108$
43. Mullan M, Crawford F, Axelman K, Houlden H, Lilius L, Winblad B, Lannfelt L: A pathogenic mutation for probable Alzheimer's disease in the APP gene at the N-terminus of beta-amyloid. Nat Genet 1992, 1:345-347

44. Davis J, Van Nostrand WE: Enhanced pathologic properties of Dutch-type mutant amyloid $\beta$-protein. Proc Natl Acad Sci U S A 1996, 93:2996-3000

45. Van Nostrand WE, Melchor JP, Cho HS, Greenberg SM, Rebeck GW: Pathogenic effects of D23N Iowa mutant amyloid $\beta$ protein. J Biol Chem 2001, 276:32860-32866

46. Brown WR: A review of string vessels or collapsed, empty basement membrane tubes. J Alzheimers Dis 2010, 21:725-739

47. Vinters HV, Natte R, Maat-Schieman ML, van Duinen SG, Hegeman-Kleinn I, Welling-Graafland C, Haan J, Roos RA: Secondary microvascular degeneration in amyloid angiopathy of patients with hereditary cerebral hemorrhage with amyloidosis, Dutch type (HCHWA-D). Acta Neuropathol 1998, 95:235-244

48. Sellal F, Wallon D, Martinez-Almoyna L, Marelli C, Dhar A, Oesterle H, Rovelet-Lecrux A, Rousseau S, Kourkoulis CE, Rosand J, DiPucchio ZY, Frosch M, Gombert C, Audoin B, Mine M, Riant F, Frebourg T, Hannequin D, Campoin D, Greenberg SM, Tournier-Lasserve E, Nicolas G: APP mutations in cerebral amyloid angiopathy with or without cortical calcifications: report of three families a literature review. J Alzheimers Dis 2017, 56:37-46

49. Bailey KR, Rustay NR, Crawley JN: Behavioral phenotyping of transgenic and knockout mice: practical concerns and potential pitfalls. ILAR J 2006, 47:124-131

50. Jakel L, Van Nostrand WE, Nicoll JAR, Werring DJ, Verbeek MM: Animal models of cerebral amyloid angiopathy. Clin Sci 2017, 131:2469-2488

51. Yang S, Smit AF, Schwartz S, Chiaromonte F, Roskin KM, Haussler D, Miller W, Hardison RC: Patterns of insertions and their covariation with substitutions in the rat, mouse, and human genomes. Genome Res 2004, 14:517-527

52. Ellenbroek B, Youn J: Rodent models in neuroscience research: is it a rat race? Dis Model Mech 2016, 9:1079-1087

53. Miller DL, Papayannopoulos IA, Styles J, Bobin SA, Lin YY, Biemann K, Iqbal K: Peptide composition of the cerebrovascular and senile plaque core amyloid deposits of Alzheimer's disease. Arch Biochem Biophys 1993, 301:41-52

54. Alonzo NC, Hyman BT, Rebeck GW, Greenberg SM: Progression of cerebral amyloid angiopathy: accumulation of amyloid-beta40 in affected vessels. J Neuropathol Exp Neurol 1998, 57:353-359

55. Paravastua AK, Leapman RD, Yau WM, Tycko R: Molecular structural basis for polymorphism in Alzheimer's $\beta$-amyloid fibril. Proc Natl Acad Sci U S A 2008, 105:18349-18354

56. Tycko R: Solid-state NMR studies of amyloid fibril structure. Annu Rev Phys Chem 2011, 62:279-299

57. Moody DM, Brown WR, Challa VR, Ghazi-Birry HS, Reboussin DM: Cerebral microvascular alterations in aging, leukoaraiosis, and Alzheimer's disease. Ann N Y Acad Sci 1997, 826:103-116

58. Brown WR, Moody DM, Thore CR, Anstrom JA, Challa VR: Microvascular changes in the white matter in dementia. J Neurol Sci 2009, 283:28-31

59. Greenberg SM, Charidimou A: Diagnosis of cerebral amyloid angiopathy: evolution of the Boston criteria. Stroke 2018, 49:491-497

60. Lesnik Oberstein SA, van dem Boom R, van Buchem MA, van Houwelingen HC, Bakker E, Vollebregt E, Ferrari MD, Breuning MH, Hann J; Dutch CADASIL Research Group: Cerebral microbleeds in CADASIL. Neurology 2001, 57:1066-1070

61. Lee JS, Kang CH, Park SQ, Choi HA, Sim KB: Clinical significance of cerebral microbleeds locations in CADASIL with R544C NOTCH3 mutation. PLoS One 2015, 10:e0125297

62. Wallays G, Nuyens D, Silasi-Mansat R, Souffreau J, Callaerts-Vegh Z, Van Nuffelen A, Moons L, D'Hooge R, Lupu F, Carmeliet P, Collen D, Dewerchin M: Notch3 Arg170Cys knock-in mice display pathologic and clinical features of the neurovascular disorder cerebral autosomal dominant arteriopathy with subcortical infarcts and leukoen-cephalopathy. Arterioscler Thromb Vasc Biol 2011, 31:2881-2888 